This is a postprint version of the following published document:

Suñé-Auñón, A., Jorge-Peñas, A., Van Oosterwyck, H.

\& Muñoz-Barrutia, A. (2016): L1-regularized

reconstruction for traction force microscopy. En 2016

IEEE 13th International Symposium on Biomedical

Imaging (ISBI) (pp. 140-144). Prague: IEEE.

DOI: $10.1109 /$ ISBI.2016.7493230

(C) 2016. IEEE. Personal use of this material is permitted. Permission from IEEE must be obtained for all other uses, in any current or future media, including reprinting/republishing this material for advertising or promotional purposes, creating new collective works, for resale or redistribution to servers or lists, or reuse of any copyrighted component of this work in other works. 


\title{
L1-REGULARIZED RECONSTRUCTION FOR TRACTION FORCE MICROSCOPY
}

\author{
Alejandro Suñé-Auñón ${ }^{1}$, Alvaro Jorge-Peñas ${ }^{2}$, Hans Van Oosterwyck ${ }^{2,3}$, Arrate Muñoz-Barrutia ${ }^{1}$ \\ ${ }^{1}$ Bioengineering and Aerospace Engineering Department, Universidad Carlos III de Madrid; Instituto de Investigación \\ Sanitaria Gregorio Marañón, 28911 Madrid, Spain \\ ${ }^{2}$ Biomechanics Section, Department of Mechanical Engineering, and ${ }^{3}$ Prometheus, Division of Skeletal Tissue Engineering, \\ KU Leuven, 3001 Leuven, Belgium
}

\begin{abstract}
Traction Force Microscopy (TFM) is a technique widely used to recover cellular tractions from the deformation they cause in their surrounding substrate. Traction recovery is an ill-posed inverse problem that benefits of a regularization scheme constraining the solution. Typically, Tikhonov regularization is used but it is well known that $\mathrm{L}_{1}$ regularization is a superior alternative to solve this type of problems. For that, recent approaches have started to explore what could be their contribution to increase the sensitivity and resolution in the estimation of the exerted tractions. In this manuscript, we adapt the $\mathrm{L}_{1}$-regularization of the curl and divergence to 2D TFM and compare the recovered tractions on simulated and real data with those obtained using Tikhonov and $\mathrm{L}_{1}$-norm regularization.
\end{abstract}

Index Terms - Traction Force Microscopy, regularization, vector operators, $\mathrm{L}_{1}$-norm.

\section{INTRODUCTION}

Force mediated cell-matrix interactions play a key role in regulating a variety of physiological and pathological processes such as wound healing, angiogenesis or cancer metastasis [1]. The quantification of these forces is becoming an extensively used tool as it provides insight on the way cells sense and react to their microenvironment.

Traction Force Microscopy (TFM) is a widespread technique to estimate the tractions exerted by adherent cells onto the extracellular matrix (ECM) through large macromolecular assemblies known as focal adhesions [2]. Those tractions are recovered from the deformations they cause in a flexible and transparent gel mimicking the ECM mechanical properties. We focus here in the study of a $2 \mathrm{D}$ setup in which the cells are cultured on the surface of the gel and only in-plane tractions are considered.

In any TFM experiment, two images of the gel must be acquired by optical microscopy: one while the adhered cells are exerting mechanical tractions (deformed gel), and the other one, after disrupting cell tractions (relaxed gel). Comparing these two images, the displacement field of the

This work was partially supported by the European Research Council (ERC) under the EU-FP7/2007-2013 through ERC Grant Agreement $n^{\circ}$ 308223, and the Spanish Ministry of Economy and Competitiveness (TEC2013-48552-C2-1-R).

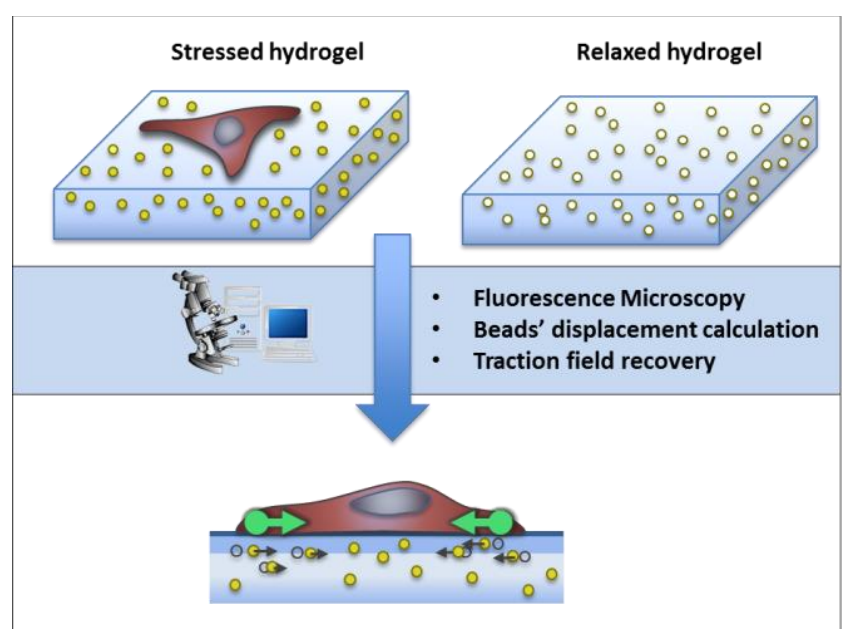

Figure 1. Schematic representation of a Traction Force Microscopy experiment.

gel is obtained and then, combined with its mechanical model to recover the cellular tractions.

Due to the optical clarity of the gels, a large number of fluorescent nanobeads embedded inside serve as references to infer the local deformation. Motion-tracking image processing techniques (i.e., particle image velocimetry, particle tracking velocimetry) are typically applied to calculate the displacements of these beads.

Due to experimental limitations, the measured displacements are usually under-sampled, which makes the mapping between the effects (gel displacements) and their originating causes (cellular tractions) non-unique. Indeed, the recovery of cellular tractions requires solving an illposed inverse problem being the solution very sensitive to the errors in the measured displacement field. To deal with this situation, the traction recovery is usually regularized to constraint the solution. The classical zeroth order Tikhonov regularization, which imposes a penalty in the $\mathrm{L}_{2}$-norm of the tractions, is commonly employed. However, this regularization causes a considerable smoothing of the solution, underestimating the traction field magnitude and spreading the recovered tractions over large areas [3].

As it is well known, $\mathrm{L}_{1}$-regularization is a superior alternative to its least squares counterpart to solve this type of problems [4]. In this manuscript, we elaborate on the very recent application of $\mathrm{L}_{1}$-regularization to $2 \mathrm{D} \operatorname{TFM}[5,6]$.

In Section 2, we present the regularized traction reconstruction of 2D TFM particularized for the $\mathrm{L}_{2}$-norm, the $\mathrm{L}_{1}$-norm and the $\mathrm{L}_{1}$-norm of the traction field curl and 
divergence. The simulations performed are described in Section 3. Then, the tractions recovered by each regularization method are compared on simulated and real data (Section 4) before providing some concluding remarks (Section 5).

\section{REGULARIZED TRACTION RECONSTRUCTION}

As indicated before, we consider the 2D TFM case. Namely, we assume that cells adhered to the surface of a linear elastic substrate exerting parallel tractions (i.e., shear tractions). Then, the gel deformation can be obtained as the direct solution of the elasticity problem:

$$
u_{j}(\boldsymbol{x})=\sum_{i} \int g_{j i}\left(\boldsymbol{x}, \boldsymbol{x}^{\prime}\right) t_{i}\left(\boldsymbol{x}^{\prime}\right) d \boldsymbol{x}^{\prime}
$$

Here, the $j$-th component $(j \in\{x, y\})$ of the displacement field $\mathbf{u}$ at spatial location $\boldsymbol{x}$ is related to the tractions $\mathbf{t}$ exerted along the $i$-th Cartesian direction $(i \in\{x, y\})$ at the location $x^{\prime}$ by the Green's function $g\left(\boldsymbol{x}, \boldsymbol{x}^{\prime}\right)$. The Green's function models the gel mechanics and it is usually given by the Boussinesq solution in $2 \mathrm{D}$ TFM [7].

Since the locations of both measured displacements and applied tractions are only obtained at discrete locations, eq. (1) can be transformed into a set of linear equations:

$$
\mathbf{u}=\mathbf{K} \cdot \mathbf{t}
$$

where $\mathbf{u}=\left[u_{x}\left(\boldsymbol{x}_{1}\right) ; \ldots ; u_{x}\left(\boldsymbol{x}_{N}\right) ; u_{y}\left(\boldsymbol{x}_{1}\right) ; \ldots ; u_{y}\left(\boldsymbol{x}_{N}\right)\right]$ is a $2 N \times 1$ column vector with $N$ being the number of locations where displacements are measured, $\mathbf{t}=$ $\left[t_{x}\left(\boldsymbol{x}_{1}^{\prime}\right) ; \ldots ;\left(\boldsymbol{x}_{M}^{\prime}\right) ; t_{y}\left(\boldsymbol{x}_{1}^{\prime}\right) ; \ldots ; t_{y}\left(\boldsymbol{x}_{M}^{\prime}\right)\right] \quad$ is a $2 M \times 1$ column vector with $M$ being the number of locations where tractions will be recovered, and $\mathbf{K}$ is the $2 N \times 2 M$ stiffness matrix given by

with

$$
\mathbf{K}=\left[\begin{array}{ll}
\mathbf{G}_{x x} & \mathbf{G}_{x y} \\
\mathbf{G}_{y x} & \mathbf{G}_{y y}
\end{array}\right]
$$

$$
\mathbf{G}_{j i}=\left[\begin{array}{ccc}
g_{j i}(1,1) & \cdots & g_{j i}(1, M) \\
\vdots & \ddots & \vdots \\
g_{j i}(N, 1) & \cdots & g_{j i}(N, M)
\end{array}\right]
$$

Since the stiffness matrix behaves as a smoothing operator, its inverse will amplify the existing errors in the calculated displacements, making the inversion of eq. (2) an ill-posed problem. To overcome this situation and stabilize the reconstruction of cellular tractions, a penalty term is commonly included and the traction recovery is reformulated as the minimization of a cost functional:

$$
\hat{\mathbf{t}}=\underset{\mathbf{t}}{\operatorname{argmin}}\left[\|\mathbf{K t}-\mathbf{u}\|_{2}^{2}+\lambda\|\mathbf{R t}\|_{p}^{p}\right]
$$

where $\|\cdot\|_{p}$ denotes the $\mathrm{L}_{\mathrm{p}}$-norm, $\mathbf{R}$ is a regularization operator and $\lambda$ is a parameter that controls the amount of regularization applied.

\section{1. $\mathrm{L}_{2}$-norm regularization}

Due to its simplicity and closed form, zero order Tikhonov regularization is still widely used in TFM. In this type of regularization, $L_{2}$ norm is used for the penalty term, and $\mathbf{R}$ is set to the identity matrix, $\mathbf{R}=\mathbf{I}$. Then, the minimization problem in eq. (5) can be analytically solved as [4]:

$$
\hat{\mathbf{t}}_{\mathrm{L}_{2}}=\left(\mathbf{K}^{\mathrm{T}} \mathbf{K}+\lambda \mathbf{I}\right)^{-1} \mathbf{K}^{\mathrm{T}} \mathbf{u}
$$

with $\mathbf{K}^{\mathrm{T}}$ being the transpose of the stiffness matrix.

\section{2. $\mathrm{L}_{1}$-norm regularization}

Alternatively, a penalty term based on the $\mathrm{L}_{1}$-norm can be used to induce the recovery of sparser traction fields. Unfortunately, we cannot obtain a closed form solution for the global minimum as for the $\mathrm{L}_{2}$ penalty. Iterative Reweighted Least Squares (IRLS) [8] can be used instead to solve the minimization problem and find $\hat{\mathbf{t}}_{\mathrm{L}_{1}}$ as was used in [5]. This algorithm approximates the $\mathrm{L}_{1}$-norm by a weighted $\mathrm{L}_{2}$-norm problem, whose weights are updated iteratively. Then, at each iteration $s$, an algebraic solution of the problem can be easily derived as approximation of the $\mathrm{L}_{1}$ norm:

$$
\hat{\mathbf{t}}_{\mathrm{L}_{1}}^{s}=\left(\mathbf{K}^{\mathrm{T}} \mathbf{K}+\lambda \mathbf{W}^{s}\right)^{-1} \mathbf{K}^{\mathrm{T}} \mathbf{u}
$$

where $\mathbf{W}^{s}$ is the weight matrix at the $s$-th iteration.

\section{3. $\mathrm{L}_{1}$-norm regularization of the curl and divergence}

Another alternative regularization scheme recently proposed for vector field denoising [9], penalizes the $\mathrm{L}_{1}$-norm of the traction field curl and divergence. Particularly, for the 2D traction fields found in TFM experiments, the curl and divergence are given by:

$$
\operatorname{curl}(\mathbf{t})=\left(\frac{\partial t_{y}}{\partial x}-\frac{\partial t_{x}}{\partial y}\right) \overrightarrow{\boldsymbol{z}} \quad \operatorname{div}(\mathbf{t})=\frac{\partial t_{x}}{\partial x}+\frac{\partial t_{y}}{\partial y}
$$

Then, the general regularization formulation in eq. (5) is transformed to:

$$
\begin{gathered}
\hat{\mathbf{t}}_{\mathrm{L}_{1, \mathrm{~cd}}}=\operatorname{argmin}_{\mathbf{t}}\left[\|\mathbf{K t}-\mathbf{u}\|_{2}^{2}+\lambda_{c}\left\|\mathbf{R}_{\mathrm{curl}} \mathbf{t}\right\|_{1}^{1}+\right. \\
\left.+\lambda_{d}\left\|\mathbf{R}_{\mathrm{div}} \mathbf{t}\right\|_{1}^{1}\right]
\end{gathered}
$$

where $\lambda_{c}, \lambda_{d}$ are the parameters that controls the amount of regularization, and $\mathbf{R}_{\text {curl }}, \mathbf{R}_{\text {div }}$ are two matrices that encodes the curl and divergence operators with central differences, respectively. Here, we have considered central differences of order three to obtain an accurate approximation of the derivatives. Also in this case, the iterative Reweighted Least Squares (IRLS) is used to solve the minimization problem and find $\hat{\mathbf{t}}_{\mathrm{L}_{1, \mathrm{~cd}}}$.

\section{EVALUATION ON SIMULATED DATA}

\subsection{Generation of the simulated data}


We have simulated point-like tractions applied along the $\mathrm{x}$ axis. We have chosen the magnitude of the simulated focal adhesions $\left(t_{g t}\right)$ to follow a Gaussian distribution:

$$
t_{g t}(\boldsymbol{x})=t_{\text {max }} e^{-\left(\frac{\left(\boldsymbol{x}-\boldsymbol{x}_{c}\right)^{2}}{2 \sigma}\right)} H(\boldsymbol{x})
$$

where $\boldsymbol{x}_{\mathrm{c}}$ is the center of the circle, $\mathrm{t}_{\max }$ is the magnitude of the simulated traction at its center $(10 \%$ of the gel Young modulus), $\sigma$ is the Gaussian standard deviation (chosen such as the magnitude of the tractions decrease a $30 \%$ of their peak magnitude at the border) and $H(\boldsymbol{x})$ is a Heaviside function (to force a null background).

We consider the simulated hydrogel to be linear, isotropic, homogeneous and elastic with a Young's modulus of $1.5 \mathrm{kPa}$ and a Poisson ratio of 0.45 . The forward equation (2) can then be applied to compute the ideal displacements and then, white Gaussian noise with a standard deviation of $0.02 \mu \mathrm{m}$ is added. The resulting noisy displacements (with a isotropic pixel size with a length of $0.25 \mu \mathrm{m}$ ) are randomly downsampled considering a density of one bead per $\mu m^{2}$. Finally, to simplify the computations, these down sampled displacements are interpolated using a non-uniform linear method to fit their initial resolution.

The optimal parameter $\lambda$ is chosen for each of the regularization methods through an exhaustive search minimizing the least squares error between the simulated and the recovered tractions.

\subsection{Error metrics}

We have evaluated the error in the recovery of the traction field within a region of interest defined by the stress footprint. This footprint has been obtained by segmenting the magnitude of the recovered tractions using an Otsu threshold.

Being $\mathbf{t}_{\mathrm{gt}}$ the simulated ground-truth traction field, and $\hat{\mathbf{t}}$ the retrieved traction field, $\mathrm{P}$ and $\mathrm{Q}$ the total number of points within the respective stress footprints, the error metrics are defined as given below.

Error in magnitude. Absolute error in the recovered traction magnitude (in percentage):

$$
e_{m}=100 \cdot \frac{\sum_{i=1}^{Q}\left|\left\|\hat{\mathbf{t}}\left(x_{i}\right)\right\|-\left\|\mathbf{t}_{g t}\left(x_{i}\right)\right\|\right|}{\sum_{i=1}^{P}\left\|\mathbf{t}_{g t}\left(\boldsymbol{x}_{i}\right)\right\|}
$$

Error in angle. Average angular error (in degrees) in the recovered stress footprint, weighted at each point by the traction magnitude:

$$
e_{\mathrm{a}}=\sum_{n=1}^{Q}\left(\frac{\|\hat{\mathbf{t}}\|_{i}}{\sum_{i=1}^{Q}\|\hat{\mathbf{t}}\|_{i}}\right) \cos ^{-1}\left(\frac{\hat{\mathbf{t}}_{i} \cdot \boldsymbol{t}_{g t}}{\|\hat{\mathbf{t}}\|_{i}\left\|\boldsymbol{t}_{g t}\right\|}\right)
$$

Error in area: Absolute error of the recovered stress footprint area (in percentage):

$$
e_{A}=100 \cdot\left|\frac{A-A_{g t}}{A_{g t}}\right|
$$

with A and $A_{g t}$ being the area of the recovered and groundtruth footprints, respectively.

\section{EXPERIMENTAL RESULTS}

\subsection{Simulated data}

We use the procedure described in Section 3.1 to generate point-like tractions of $3 \mu \mathrm{m}$ radius, applied along the $\mathrm{x}$-axis and the corresponding noisy displacements for three conditions: an isolated and two focal adhesions separated by 1.5 and $3 \mu \mathrm{m}$. For each condition, ten realizations are considered.

Figure 2 (a) shows an example of a simulated traction field for two circular traction patches with a separation of $1.5 \mu \mathrm{m}$. The corresponding ideal and noisy displacements are shown in Figures 2 (b) and (c), respectively. The magnitude and orientation of the recovered traction field using the different regularizations are shown in Figures 2 (df). We observe that the zero order Tikhonov regularization collapses the point-like tractions in a unique stress footprint while the $\mathrm{L}_{1}$-regularizations recover both of them. In terms of the magnitude, observe that $\mathrm{L}_{2}$-norm regularization tends to underestimate while the $\mathrm{L}_{1}$-norm regularization slightly overestimates, compared with the estimation performed by the $\mathrm{L}_{1}$-norm regularization of the curl and divergence. Note also how the $\mathrm{L}_{1}$-norm regularization recovers nearly no background noise while the $\mathrm{L}_{2}$-norm regularization generates a significant amount of it. The $\mathrm{L}_{1}$-nrom regularization of the curl and divergence generates a certain amount of noise due mainly to the derivatives computation. In terms of the traction orientations, $\mathrm{L}_{1}$-norm regularization recovers the cleanest map being the nosiest the one obtained by the $\mathrm{L}_{1}$-norm regularization of the curl and divergence.

Table 1 presents the computed error metrics. Overall, $\mathrm{L}_{1}$-norm regularization performs best having the $\mathrm{L}_{1}$-norm regularization of the curl and divergence slightly smaller magnitude error.

\subsection{Real data}

In this section, we present an example of traction recovery on a real cell with the different regularization approaches. Fluorescent polystyrene microbeads $(0.2 \mu \mathrm{m}$ in diameter $)$ were embedded in a $\sim 90 \mu \mathrm{m}$ thick polyacrylamide hydrogel with a Young's modulus of $1.3 \mathrm{kPa}$ and a Poisson ratio of 0.45 . Human umbilical vein endothelial cells (HUVEC) expressing the Green fluorescent protein were cultured on the surface of hydrogel. Images of the cells (see Figure 3 (a)) and the polyacrylamide gel (see Figure 3 (b)) containing HUVEC were acquired at multiple locations of the surface of the stressed hydrogel with a $40 x$ dry objective (NA $=0.95$ ) mounted in an Olympus FV1000-IX81 FluoView LaserScanning Confocal microscope. After imaging, detergent was added to the culture medium. Once the cells were completely removed and the gel had returned to the unstrained state, new images of the fluorescent beads were taken at the previously recorded locations. 


\begin{tabular}{|c|c|c|c|c|c|c|c|c|c|}
\hline \multirow[b]{2}{*}{ Errors } & \multicolumn{3}{|c|}{ One Focal Adhesion } & \multicolumn{3}{|c|}{$1.5 \mu \mathrm{m}$ separation } & \multicolumn{3}{|c|}{$3 \mu \mathrm{m}$ separation } \\
\hline & $\mathbf{L}_{2}$ & $\mathbf{L}_{1}$ & $\mathrm{~L}_{1, \mathrm{~cd}}$ & $\mathrm{~L}_{2}$ & $\mathrm{~L}_{1}$ & $\mathbf{L}_{1, \mathrm{~cd}}$ & $\mathbf{L}_{2}$ & $\mathbf{L}_{1}$ & $\mathbf{L}_{1, \mathrm{~cd}}$ \\
\hline Mag (\%) & $2.02 \pm 0.14$ & $1.71 \pm 0.16$ & $1.61 \pm 0.28$ & $1.95 \pm 0.14$ & $1.69 \pm 0.09$ & $1.57 \pm 0.17$ & $2.00 \pm 0.07$ & $1.73 \pm 0.11$ & $1.46 \pm 0.12$ \\
\hline Ang $\left({ }^{\circ}\right)$ & $4.09 \pm 1.06$ & $(4.60 \pm 1.43) 10^{-5}$ & $9.02 \pm 3.52$ & $4.17 \pm 1.03$ & $(3.22 \pm 1.1) 10^{-5}$ & $7.21 \pm 1.82$ & $3.96 \pm 0.64$ & $0.03 \pm 0.01$ & $8.68 \pm 1.76$ \\
\hline Area (\%) & $11.97 \pm 2.42$ & $3.24 \pm 1.27$ & $13.83 \pm 5.83$ & $15.91 \pm 2.48$ & $1.51 \pm 0.22$ & $12.69 \pm 4.56$ & $13.17 \pm 1.59$ & $2.15 \pm 0.92$ & $9.35 \pm 3.26$ \\
\hline
\end{tabular}

Table 1. Quantitative comparison of the recovered tractions for $\mathrm{L}_{2}$-norm, $\mathrm{L}_{1}$-norm and $\mathrm{L}_{1}$-norm regularization of the curl and divergence $\left(\mathrm{L}_{1, \mathrm{~cd}}\right)$. Ten realizations were used for each case.

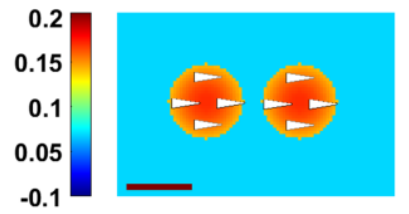

(a)

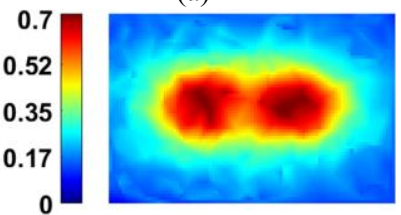

(c)

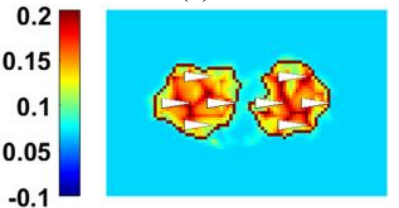

(e)

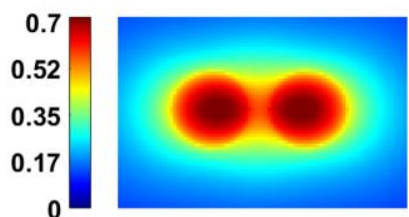

(b)
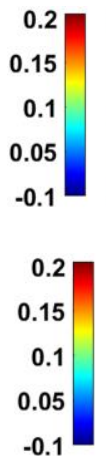

$-0.1$

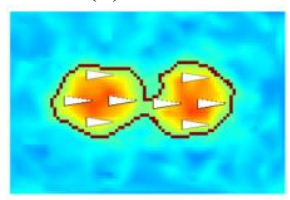

(d)

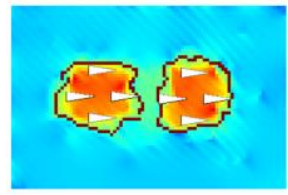

(f)

Figure 2. Simulated data. (a) Magnitude (in $\mathrm{kPa}$ ) and orientation (in angles) of a traction field applied in the $\mathrm{x}$ axis simulating two circular focal adhesions of $3 \mu \mathrm{m}$ of radius, $1.5 \mu \mathrm{m}$ of separation and a peak magnitude of $10 \%$ of the gel Young Modulus; (b) Magnitude (in $\mu \mathrm{m}$ ) of the displacement field calculated from the simulated tractions; (c) Noisy displacements used for the traction recovery step; (d-f) Magnitude and orientation of the recovered traction field obtained with zero order Tikhonov, $\mathrm{L}_{1}$-norm and $\mathrm{L}_{1}$-norm regularization of the curl and divergence, respectively. The scale bar represents $5 \mu \mathrm{m}$. The brown line corresponds to Otsu thresholding.

Figure 4 (a) shows for a zoomed region of the cell, the estimated displacements and (b-d) the recovered tractions magnitude and orientations for the different regularizations. As expected, the zero order Tikhonov regularization when compared to their $\mathrm{L}_{1}$ counterparts smoothens the solution, estimating lower traction field magnitudes and spreading the recovered tractions over large areas.

\section{CONCLUSION}

In this manuscript, we have compared the performance of different regularization schemes (Tikhonov, $\mathrm{L}_{1}$-norm, $\mathrm{L}_{1}$ norm of the curl and divergence) when applied to constraint the traction reconstruction in $2 \mathrm{D}$ TFM experiments. $\mathrm{L}_{1}$ regularization of the tractions reduces the background noise recovering the traction magnitude with a smaller error than Tikhonov regularization. More importantly, $\mathrm{L}_{1}$ regularization promotes the recovery of sparser traction fields; thus, better reproducing the nature of cellular tractions, which are mainly localized at few discrete clusters of adhesion sites.

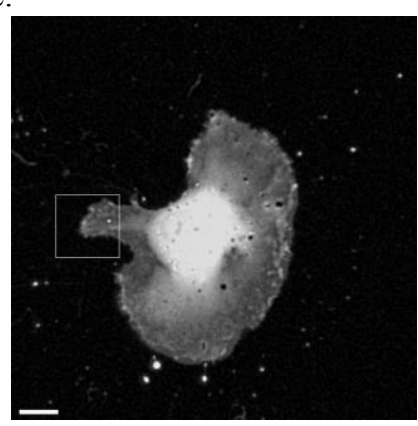

(a)

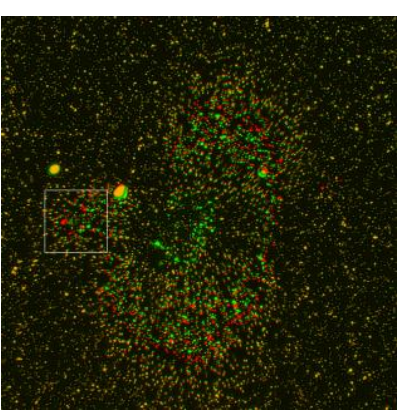

(b)
Figure 3. Bead displacements induced by a real cell. The beads of the unstressed and stressed hydrogels have been pseudo-colored in red and green, respectively; therefore, beads are colored yellow when not displaced. Additionally, the contrast of the pseudo-color image has been modified to highlight the areas with bead displacements. The scale bar represents $20 \mu \mathrm{m}$.

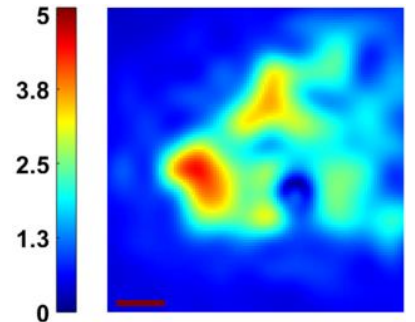

(a)

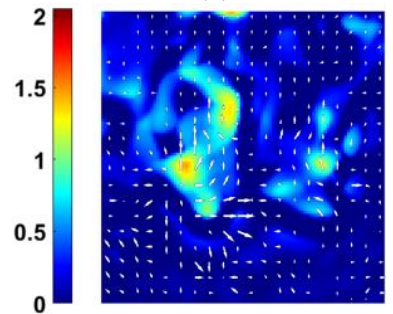

(c)

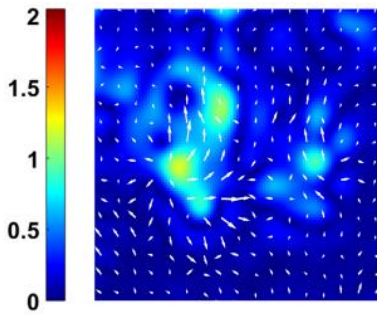

(b)

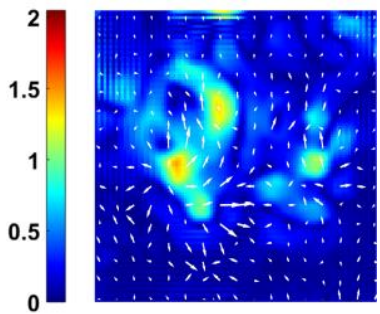

(d)
Figure 4. Experimental data (a) Estimated displacements (in $\mu \mathrm{m}$ ) recovered from the bead positions. Recovered tractions magnitude (in $\mathrm{kPa}$ ) and orientations (in angles) using: (b) zero order Tikhonov regularization; (c) $\mathrm{L}_{1}$-norm regularization; (d) $\mathrm{L}_{1}$-norm regularization of the curl and divergence. The images correspond to the square box shown in Figure 3 (b). The scale bar represents $5 \mu \mathrm{m}$. 


\section{REFERENCES}

[1] D. E. Discher, P. Janmey, Y. L- Wang, "Tissue cells feel and respond to the stiffness of their substrate," Science, vol. 310, no. 5751, pp. 1139-43, 2005.

[2] J. P. Butler, I.M. Tolic'-Nørrelykke, B. Fabry, J.J. Fredberg, "Traction fields, moments, and strain energy that cells exert on their surroundings", American Journal of Physiology-Cell Physiology, vol. 282, no. 3, pp. C595- C605, 2002.

[3] J. Westerweel, D. Dabiri and M. Gharib, "The effect of a discrete window offset on the accuracy of cross-correlation analysis of digital PIV recordings," Experiments in Fluids, vol. 23, no. 1, pp. 20-28, 1997.

[4] E. J. Candes, J. Romberg and T. Tao, "Robust uncertainty principles: Exact signal reconstruction from highly incomplete frequency information," IEEE Transactions on Information Theory, vol. 52, no. 2, pp. 489-509, 2006.

[5] S. J. Han, Y. Oak, A. Grisman, G. Danuser, "Traction Microscopy to identify force modulation in subresolution adhesions", Nature Methods, vol. 12, no. 7, pp. 653-656, 2015.

[6] J. Bohr, G. Singla-Buxarrais, R. Vincent, X. Trepat, "Compressed sensing traction force microscopy", Acta Biomaterialia, vol. 26, pp. 286-294, 2015

[7] L. Landau, E. Lifshitz, "Theory of elasticity: course of theoretical physics", vol. 7, Oxford: Pergamon Press, 1970.

[8] J. A. Scales, A. Gerztenkorn, S. J. Treitel, "Fast Lp solution of large, sparse, linear systems: Application to seismic travel time tomography", Journal of Computational Physics, vol. 75, pp. 314-333, 1988.

[9] P. D. Tafti, M. Unser, "On regularized reconstruction of vector fields", IEEE Transactions on Image Processing, vol. 20, no. 11, pp. 3163-3178, 2011. 